Pacific

Journal of

Mathematics

ON THE MODULI SPACE OF THE SCHWARZENBERGER BUNDLES

Paolo Cascini

Volume $205 \quad$ No. 2

August 2002 


\title{
ON THE MODULI SPACE OF THE SCHWARZENBERGER BUNDLES
}

\author{
Paolo Cascini
}

For any odd $n$, we prove that the coherent sheaf $\mathcal{F}_{A}$ on $\mathbb{P}_{\mathbb{C}}^{n}$, defined as the cokernel of an injective map $f: \mathcal{O}_{\mathbb{P}^{n}}{ }^{\oplus 2} \rightarrow$ $\mathcal{O}_{\mathbb{P}^{n}}(1)^{\oplus(n+2)}$, is Mumford-Takemoto stable if and only if the map $f$ is stable, when considered as a point of the projective space $\mathbb{P}\left(\operatorname{Hom}\left(\mathcal{O}_{\mathbb{P} n}{ }^{\otimes 2}, \mathcal{O}_{\mathbb{P} n} \otimes(n+2)\right)^{*}\right)$ under the action of the reductive group $\mathrm{SL}(2) \times \mathrm{SL}(n+2)$. This proves a particular case of a conjecture of J.-M.Drezet and it implies that a component of the Maruyama scheme of the semi-stable sheaves on $\mathbb{P}^{n}$ of rank $n$ and Chern polynomial $(1+t)^{n+2}$ is isomorphic to the Kronecher moduli $N(n+1,2, n+2)$, for any odd $n$. In particular, such scheme defines a smooth minimal compactification of the moduli space of the rational normal curves in $\mathbb{P}^{n}$, that generalizes the construction defined by G. Ellinsgrud, R. Piene and S. Strømme in the case $n=3$.

\section{Introduction.}

Let us consider all the exact sequences:

$$
0 \longrightarrow I^{*} \otimes \mathcal{O}_{\mathbb{P}(V)} \stackrel{f_{A}}{\longrightarrow} W^{*} \otimes \mathcal{O}_{\mathbb{P}(V)}(1) \longrightarrow \mathcal{F}_{A} \longrightarrow 0
$$

where $W, V$ and $I$ are complex vector spaces of dimension $m+k, n+1$ and $k$ respectively, $f_{A}$ is an injective morphism of sheaves canonically induced by a linear map $A \in \mathbb{P}\left(\operatorname{Hom}(W, I \otimes V)^{*}\right)\left(=\mathbb{P}\left(\operatorname{Hom}\left(I^{*} \otimes \mathcal{O}_{\mathbb{P}(V)}, W^{*} \otimes \mathcal{O}_{\mathbb{P}(V)}(1)\right)^{*}\right)\right)$ and $\mathcal{F}_{A}=$ Coker $f_{A}$ is a coherent sheaf of rank $m$ over the projective space $\mathbb{P}(V)\left(=\left(V^{*} \backslash\{0\}\right) / \mathbb{C}^{*}\right)$.

In particular, if $n=m$ and if the degeneracy locus of $f_{A}$ is empty, then $\mathcal{F}_{A}$ is a vector bundle of rank $n$ on $\mathbb{P}^{n}$, called Steiner bundle. In $[\mathbf{G K Z}]$ it is shown that $A$, considered as a multidimensional matrix of size $(n+k) \times$ $k \times(n+1)$, defines a Steiner bundle $\mathcal{F}_{A}$ if and only if its hyperdeterminant does not vanish.

In [AO], the authors give a complete description of the moduli space $S_{n, k}$ of the Steiner bundles on $\mathbb{P}^{n}$ : Such moduli space can be considered as an open subset of the categorical quotient:

$$
\mathcal{M}_{n, m, k}=\mathbb{P}\left(\operatorname{Hom}(W, I \otimes V)^{*}\right) / /(\mathrm{SL}(I) \times \mathrm{SL}(W)) .
$$


with $n=m$. It is known that $\mathcal{M}_{n, m, k}$ is canonically isomorphic to the Kronecker module $N(n+1, k, m+k)$ defined as the quotient $\mathbb{G}(W, I \otimes V) / / \mathrm{SL}(I)$ : The isomorphism is given by considering the image $T_{A}:=A(W)$ of the linear map $A: W \rightarrow I \otimes V$. Such modules are extensively described in [Dr1] and [Dr2]. In particular we have:

Theorem 1.1. Let $A \in \mathbb{P}\left(\operatorname{Hom}(W, I \otimes V)^{*}\right)$ and $T=A(W) \subseteq I \otimes V$. The following are equivalent:

(1) $A$ is semi-stable (resp. stable) under the action of $\mathrm{SL}(I) \times \mathrm{SL}(W)$;

(2) $T \in \mathbb{G}(m+k, I \otimes V)$ is semi-stable (resp. stable) under the action of $\mathrm{SL}(I)$

(3) for any nonempty subspace $I^{\prime} \subsetneq I$

$$
\frac{\operatorname{dim} T^{\prime}}{\operatorname{dim} I^{\prime}} \leq \frac{\operatorname{dim} T}{\operatorname{dim} I} \quad(\text { resp. }<)
$$

where $T^{\prime}=\left(I^{\prime} \otimes V\right) \cap T$.

In general, if $m \geq n$, every element $A$ of $\mathcal{M}_{n, m, k}$ determines a coherent sheaf $\mathcal{F}_{A}$ on $\mathbb{P}^{n}=\mathbb{P}(V)$ of rank $m$ : In fact, every $A: W \rightarrow I \otimes V$ induces a morphism $f_{A}: I^{*} \otimes \mathcal{O}_{\mathbb{P}^{n}} \rightarrow W^{*} \otimes \mathcal{O}_{\mathbb{P}^{n}}(1)$, as in (1). We will call Steiner bundle of rank $m$, a vector bundle $\mathcal{F}_{A}$ contained in the sequence (1) even when $m \geq n$. Such bundles defines a moduli space $S_{n, m, k}$, that is an open subset of the projective variety $\mathcal{M}_{n, m, k}$.

Important examples of rank $n$ Steiner bundles are the Schwarzenberger bundles $[\mathbf{S c h w}]$, defined by the morphism

$$
f_{A}=\left(\begin{array}{cccccc}
x_{0} & x_{1} & \ldots & x_{n} & & \\
& \ddots & \ddots & & \ddots & \\
& & x_{0} & x_{1} & \ldots & x_{n}
\end{array}\right)^{t} \in \mathbb{P}\left(\operatorname{Hom}\left(I^{*} \otimes \mathcal{O}_{\mathbb{P}^{n}}, W^{*} \otimes \mathcal{O}_{\mathbb{P}^{n}}(1)\right)^{*}\right) .
$$

The set of equivalence classes of these bundles is in one-one correspondence with the variety $S_{n}$ of the rational normal curves. In fact if $W(S)=$ $\left\{H \in\left(\mathbb{P}^{n}\right)^{*} \mid h^{0}\left(\left(\mathcal{F}_{A}^{*} \otimes \mathcal{O}_{\mathbb{P}^{n}}(1)\right)_{\mid H}\right) \neq 0\right\}$ is the scheme of the unstable hyperplanes of a Steiner bundle of rank $n \mathcal{F}_{A}$, then $\mathcal{F}_{A}$ is a Schwarzenberger bundle if and only if $W(S)$ is a rational normal curve in $\left(\mathbb{P}^{n}\right)^{*}$ (see $[\mathbf{V}]$ ).

In particular, if $k=2$, all the indecomposable Steiner bundles are Schwarzenberger bundles (see $[\mathbf{D K}]$ ), and thus $S_{n} \simeq S_{n, 2} \simeq \mathbb{P} \mathrm{GL}(n+1) / \mathrm{SL}(2)$. In this paper we will consider exactly this case. In fact we will show that, if $k=2$ and $m$ is odd, then $A \in \mathbb{P}\left(\operatorname{Hom}(W, I \otimes V)^{*}\right)$ is stable if and only if the correspondent coherent sheaf $\mathcal{F}_{A}$ is $\mu$-stable. This will imply the following:

Theorem 1.2. $\mathcal{M}_{n, m, 2}$ is isomorphic to the connected component of the Maruyama moduli space $\mathcal{M}_{\mathbb{P} n}\left(m, c_{1}, \ldots, c_{n}\right)$ containing the Steiner bundles. Such component is smooth and irreducible. 
This result gives an affirmative answer to a particular case of a question queried by J.-M. Drezet [Dr3]. Before that, R.M. Miro-Roig and G. Trautmann had proved a similar result in the case $n=3, k=2$ and $m=3$ [MT].

Moreover the variety $\mathcal{M}_{n, n, 2}$ defines a smooth compactification of the moduli space of the rational normal curves in $\mathbb{P}^{n}$ for any odd $n$ (this result is proved in $[\mathbf{D r} 2]$ and in $[\mathbf{E S}])$. In fact, such construction generalizes the one given in [EPS], defined as the variety of nets of quadrics defining twisted cubics.

From a topological point of view, $[\mathrm{Dr} 2]$ provides a method to compute the Betti numbers of $\mathcal{M}_{n, m, 2}$ (see also [C] for further details).

I would like to thank V. Ancona and G. Ottaviani for many fruitful discussions and the referee for his very helpful comments.

\section{Preliminares.}

Let $W, V$ and $I$ be complex vector spaces of dimension $m+2, n+1$ and 2 respectively, with $2+m \leq 2(n+1)$ and let us define $X=\mathbb{P}\left(\operatorname{Hom}(W, I \otimes V)^{*}\right)$.

For any $\omega \in I$ we define $R_{\omega}=\omega \otimes V \subseteq I \otimes V$ : By Theorem 1.1 we have that an injective linear map $A \in X$ is semi-stable (resp. stable) under the action of $\mathrm{SL}(I) \times \mathrm{SL}(W)$ if and only if, for any $\omega \in I$,

$$
\left.\operatorname{dim} R_{\omega} \cap T_{A} \leq \frac{m+2}{2} \quad \text { (resp. }<\right),
$$

where, we remind, $T_{A}$ is the image of $W$ by $A$ (the arithmetic assumption over $n$ and $m$ guarantees that $X^{s s}$ is not empty).

Let $D(A)$ denote the degeneracy locus of $f_{A}$, i.e., the set of all the points $x \in \mathbb{P}^{n}$ such that $\operatorname{rank}\left(\left(f_{A}\right)_{x}: I^{*} \otimes \mathcal{O}_{\mathbb{P}^{n}, x} \rightarrow W^{*} \otimes \mathcal{O}_{\mathbb{P}^{n}, x}(1)\right) \leq 1$, then for any $j \in \mathbb{N}$ we construct the subsets:

$$
\begin{aligned}
S^{j} & =\left\{A \in X^{s s} \mid \exists \omega \in I \text { such that } \operatorname{dim} R_{\omega} \cap T_{A} \geq j+m-n\right\} \quad \text { and } \\
\widetilde{S}^{j} & =\left\{A \in X^{s s} \mid \operatorname{dim} D(A) \geq j-2\right\} .
\end{aligned}
$$

These subsets canonically define two filtrations of $X$ :

$$
\begin{aligned}
& \emptyset=S^{j_{0}+1} \subseteq S^{j_{0}} \subseteq \cdots \subseteq S^{2} \subseteq S^{1}=X^{s s} \\
& \emptyset \subseteq \cdots \subseteq \widetilde{S}^{j_{0}+1} \subseteq \widetilde{S}^{j_{0}} \subseteq \cdots \subseteq \widetilde{S}^{2} \subseteq \widetilde{S}^{1}=X^{s s}
\end{aligned}
$$

where $j_{0}=\left[\frac{m+3}{2}\right]+n-m([x]$ denotes the integer part of $x \in \mathbb{Q})$.

It results $S^{j_{0}}=X^{s s} \backslash X^{s}$ and in particular it is empty if $m$ is odd. Furthermore we have:

\section{Theorem 2.1.}

(1) $S^{j} \subseteq \widetilde{S}^{j} \subseteq S^{j-1}$ for any $j \geq 2$;

(2) $S^{2}=\widetilde{S}^{2}$; 
(3) $S^{1}=\widetilde{S}^{1}=X^{s s}$.

In particular such subsets define a unique $G$-invariant filtration:

$$
\begin{array}{r}
\emptyset=S^{j_{0}+1} \subseteq \widetilde{S}^{j_{0}+1} \subseteq S^{j_{0}} \subseteq \widetilde{S}^{j_{0}} \subseteq \cdots \\
\cdots \subseteq S^{3} \subseteq \widetilde{S}^{3} \subseteq S^{2}=\widetilde{S}^{2} \subseteq S^{1}=\widetilde{S}^{1}=X^{s s} .
\end{array}
$$

Before proving the theorem, we remind the following known lemma:

Lemma 2.2. Let $F$ be a vector bundle of rank $f$ on a smooth projective variety $X$ such that $c_{f-k+1}(F) \neq 0$ and let $\phi: \mathcal{O}_{X}^{k} \longrightarrow F$ be a morphism with $k \leq f$. Then the degeneracy locus $D(\phi)=\left\{x \in X \mid \operatorname{rank}\left(\phi_{x}\right) \leq k-1\right\}$ is nonempty and codim $D(\phi) \leq f-k+1$.

Proof of Theorem 2.1.

(1) Let $A \in S^{j}$, then there exists $\omega \in I$ such that $\operatorname{dim} R_{\omega} \cap T_{A} \geq j+m-n$ and thus $\omega$ defines a morphism of sheaves: $\widetilde{f_{A}}: \mathcal{O}_{\mathbb{P}^{n}} \rightarrow \mathcal{O}_{\mathbb{P}^{n}}(1)^{n-j+2}$. The degeneracy locus of $\widetilde{f}_{A}$ is contained in $D(A)$ and by Lemma 2.2 , since $c_{n-j+2}\left(\mathcal{O}_{\mathbb{P}^{n}}(1)^{n-j+2}\right) \neq 0$ if $j \geq 2$, it follows that $\operatorname{dim} D(A) \geq$ $j-2$, i.e., $S^{j} \subseteq \widetilde{S}^{j}$ for any $j \geq 2$.

Let now $A \in \widetilde{S}^{j}$ and let us denote by $D_{0}(A)$ the variety of all the points $x \in \mathbb{P}^{n}$ such that $\operatorname{rank}\left(f_{A}\right)_{x}=0$. We consider first the case $D_{0}(A) \subsetneq D(A)$ : Each point $x \in \mathbb{P}^{n}$ naturally defines an evaluation map $\eta_{x}: I \otimes V \rightarrow \mathbb{P}\left(I^{*}\right)$. Thus we can define $\pi: D(A) \backslash D_{0}(A) \rightarrow \mathbb{P}\left(I^{*}\right)$ where $\pi(x)$ is the only point of $\eta_{x}\left(T_{A}\right)$ and, since $\operatorname{dim} D(A) \backslash D_{0}(A) \geq$ $j-2$, there exists $\omega \in I$ such that $\operatorname{dim} \pi^{-1}([\omega]) \geq j-3$.

Let $R_{\omega}^{\prime}=\left\{f \in I \otimes V \mid \eta_{x}(f)=[\omega]\right.$ in $\mathbb{P}\left(I^{*}\right)$ for any $\left.x \in \pi^{-1}([\omega])\right\}$ : In order to compute the dimension of $R_{\omega}^{\prime}$ we consider $p_{1}, \ldots, p_{j-2} \in$ $\pi^{-1}([\omega])$ not contained in a linear subspace $\mathbb{P}^{j-4} \subseteq \mathbb{P}^{n}$ : Such points define a linear system of $j-2$ linearly independent equations whose solutions are contained in $R_{\omega}^{\prime}$ and thus we have $\operatorname{dim} R_{\omega}^{\prime} \leq 2(n+1)-$ $(j-2)=2 n+4-j$.

Since $T_{A}, R_{\omega} \subseteq R_{\omega}^{\prime}$, we have that

$\operatorname{dim} T_{A} \cap R_{\omega} \geq \operatorname{dim} T+\operatorname{dim} R_{\omega}-\operatorname{dim} R_{\omega}^{\prime} \geq j+m-n-1$, i.e., $A \in S^{j-1}$.

If $D_{0}(A)=D(A)$, then it can be similarly proven that for any $\omega \in I$, $\operatorname{dim} R_{\omega}^{\prime} \leq 2 n+3-j$ and thus $A \in S^{j} \subseteq S^{j-1}$.

(2) We have already proven that $S^{2} \subseteq \widetilde{S}^{2}$. Let now $A \in \widetilde{S}^{2}$. As before, we can suppose $D_{0}(A) \subsetneq D(A)$.

Let $x \in D(A) \backslash D_{0}(A)$ and $\omega \in I$ such that $\eta_{x}\left(T_{A}\right)=\{[\omega]\}$. If $R_{\omega}^{\prime}=$ $\left\{f \in I \otimes V \mid \eta_{x}(f)=[\omega]\right.$ in $\left.\mathbb{P}\left(I^{*}\right)\right\}$, then $\operatorname{dim} R_{\omega}^{\prime}=2 n+1: T_{A}, R_{\omega} \subseteq R_{\omega}^{\prime}$ and thus $\operatorname{dim} T_{A} \cap R_{\omega} \geq(m+2)+(n+1)-(2 n+1)=m-n+2$, i.e., $A \in S^{2}$.

(3) Both the equalities are trivial. 
Remark 2.3. In general $S^{i} \neq \widetilde{S}^{i}$ : Let us consider, for instance, $n=m=3$ and

$$
f_{A}=\left(\begin{array}{ccccc}
0 & 0 & x_{0} & x_{1} & x_{2} \\
x_{0} & x_{1} & 0 & 0 & x_{3}
\end{array}\right)^{t} .
$$

Since $D(A)=\left\{\left(0: 0: t_{1}: t_{2}\right)\right\} \simeq \mathbb{P}^{1}, A \in \widetilde{S}^{3} ;$ but $S^{3}=\emptyset$ (see also Proposition 2.5).

Corollary 2.4. If $m$ is odd and $A \in X^{s}=X^{s s}$ then $\operatorname{codim} D(A) \geq \frac{m+1}{2}$. If $m$ is even and $A \in X^{s s}$ (resp. $\left.X^{s}\right)$ then $\operatorname{codim} D(A) \geq \frac{m}{2}$ (resp. $>$ ).

Proof. It suffices to notice that the previous theorem implies that $\widetilde{S}^{j_{0}+1}=\emptyset$ and that $S^{j_{0}}$ is the set of the properly semi-stable points of $X$.

Proposition 2.5. If $m$ is odd, $A \in X$ is stable and $\operatorname{codim} D(A)=\frac{m+1}{2}$, where $t=\frac{m+1}{2}$, then, up to the action of $\mathrm{SL}(I) \times \mathrm{SL}(W) \times \mathrm{SL}(V)$, we have

$$
f_{A}=\left(\begin{array}{ccccccc}
x_{0} & \ldots & x_{t-1} & 0 & \ldots & 0 & x_{t} \\
0 & \ldots & 0 & x_{0} & \ldots & x_{t-1} & x_{t+1}
\end{array}\right)^{t} .
$$

Proof. By the Proof of Theorem 2.1 we have that for any $\omega \in I, \operatorname{dim}(\omega \otimes V) \cap$ $T_{A} \geq t$, where, as before, $T_{A}$ is the image of $A$ as a subspace of $I \otimes V$, and in fact, by Theorem 1.1 and since $A$ is stable, it results $\operatorname{dim}(\omega \otimes V) \cap T_{A}=t$.

Thus we have, up to a change of basis,

$$
f_{A}=\left(\begin{array}{ccccccc}
f_{0} & \ldots & f_{t-1} & 0 & \ldots & 0 & f_{t} \\
0 & \ldots & 0 & g_{0} & \ldots & g_{t-1} & g_{t}
\end{array}\right)^{t},
$$

where $\left\langle f_{0}, \ldots, f_{t}\right\rangle$ and $\left\langle g_{0}, \ldots, g_{t}\right\rangle$ are subspaces of $V$ of dimension $t+1$.

It is easily checked that $D(A)=V\left(f_{0}, \ldots, f_{t}\right) \cup V\left(g_{0}, \ldots, g_{t}\right) \cup V\left(f_{0}, \ldots\right.$, $\left.f_{t-1}, g_{0}, \ldots, g_{t-1}\right)$ and since $\operatorname{codim} D(A)=t$, it must be $\operatorname{codim} V\left(f_{0}, \ldots\right.$, $\left.f_{t-1}, g_{0}, \ldots, g_{t-1}\right)=t$ : This implies that $\left\langle f_{0}, \ldots, f_{t-1}\right\rangle=\left\langle g_{0}, \ldots, g_{t-1}\right\rangle$ and therefore we can assume $g_{i}=f_{i}$ for any $i=0, \ldots, t-1$.

Moreover $g_{t} \notin\left\langle f_{0}, \ldots, f_{t}\right\rangle$ otherwise, up to the action of $\mathrm{SL}(I) \times \mathrm{SL}(W)$, it would be

$$
f_{A}=\left(\begin{array}{ccccccc}
f_{0} & \ldots & f_{t-1} & 0 & \ldots & 0 & f_{t} \\
0 & \ldots & 0 & f_{0} & \ldots & f_{t-1} & 0
\end{array}\right)^{t}
$$

and by Theorem 1.1, $A$ would not be stable, because there would exist a vector $\omega \in I$ such that $\operatorname{dim}(\omega \otimes V) \cap T_{A}=t+1$. Therefore $f_{0}, \ldots, f_{t}$ are linearly independent and we can suppose $f_{i}=x_{i}$ for some basis $\left\{x_{0}, \ldots, x_{n}\right\}$ of $V$.

\section{Proof of Theorem 1.2.}

For any coherent sheaf $\mathcal{E}$ of rank $r$ on $\mathbb{P}^{n}, \mathcal{E}_{N}$ will denote the normalized sheaf of $\mathcal{E}$, i.e., $\mathcal{E}_{N}=\mathcal{E}\left(t_{0}\right)$ where $t_{0} \in \mathbb{Z}$ is such that $-r<c_{1}\left(\mathcal{E}\left(t_{0}\right)\right) \leq 0$. 
Moreover hd $(\mathcal{E})$ will be the homological dimension of $\mathcal{E}$ (cf. [OSS]) and $S(\mathcal{E})$ the singular locus of $\mathcal{E}$, i.e., $S(\mathcal{E})=\left\{x \mid \operatorname{dim} \mathcal{E}_{x}>r\right\}$.

In this section we will only consider sheaves $\mathcal{F}_{A}$ of odd rank $m$, i.e., such that $\left(c_{1}\left(\mathcal{F}_{A}\right), m\right)=1$. Hence the Mumford-Takemoto stability (also said $\mu$ stability) of these sheaves coincides with their Gieseker stability. Moreover $\mathcal{F}_{A}$ is stable if and only if it is semi-stable. Thus, before proceeding with the proof of Theorem 1.2, we are interested to study the relation between the G.I.T. stability of maps and the $\mu$-stability of their cokernels.

In fact we have:

Theorem 3.1. Let $k=2$ and $m \in \mathbb{N}$ odd. Then the following are equivalent:

(1) $T_{A} \in \mathbb{G}(m+2, I \otimes V)$ is G.I.T. stable;

(2) $\mathcal{F}_{A}$ is $\mu$-stable.

The main tool needed to prove the theorem is the following lemma:

Lemma 3.2. Let $A \in \mathbb{P}\left(\operatorname{Hom}(W, I \otimes V)^{*}\right)$ be a stable map, then

$$
\mathrm{H}^{0}\left(\left(\wedge^{r} \mathcal{F}_{A}\right)_{N}^{* *}\right)=0
$$

for any $r=1, \ldots, m-1$.

Later on, we will show that the vanishing of the cohomology groups in (2) will imply the $\mu$-stability of the sheaf $\mathcal{F}_{A}$.

Before proceeding with the proof of Lemma 3.2, we want to recall some facts that will be useful during the proof: Although many of these results are well-known, we report them for completeness.

For the proof of the following two propositions, see [HL] Prop. 1.1.6 and Prop. 1.1.10:

Proposition 3.3. Let $E$ be a coherent sheaf of codimension $c$ on a smooth projective variety $Z$. Then the sheaves $\mathcal{E x t}^{q}\left(E, \omega_{Z}\right)$ are supported on $\operatorname{Supp}(E)$ and $\mathcal{E} x t^{q}\left(E, \omega_{Z}\right)=0$ for all $q<c$.

Proposition 3.4. Let $E$ be a coherent sheaf on a smooth projective variety $Z$. Then the following conditions are equivalent:

(1) $\operatorname{codim}\left(\mathcal{E} x t^{q}\left(E, \omega_{Z}\right)\right) \geq q+1$ for any $q \geq 1$;

(2) the canonical map $E \rightarrow E^{* *}$ is injective.

Similarly, the following are equivalent:

(1) $\operatorname{codim}\left(\mathcal{E} x t^{q}\left(E, \omega_{Z}\right)\right) \geq q+2$ for any $q \geq 1$;

(2) $E$ is the dual of a coherent sheaf;

(3) $E$ is reflexive.

We will also need: 
Lemma 3.5. Let $s$ be a section of a vector bundle $E$ of rank $r$ on an algebraic variety $Z$ and let $Z_{0}$ be the zero locus of $s$. If $Z_{0}$ is of codimension $r^{\prime} \leq r$ then the Koszul complex associated to $s$ induces an exact sequence of the first $r^{\prime}+1$ terms:

$$
0 \rightarrow \operatorname{det} E^{*} \rightarrow \wedge^{r-1} E^{*} \rightarrow \cdots \rightarrow \wedge^{r-r^{\prime}} E^{*} .
$$

Proof. By Bertini theorem, it easily follows that there exists a complete intersection subvariety $Z^{\prime} \subseteq Z$ of codimension $r^{\prime}$ and containing $Z_{0}$.

It is enough to prove the lemma after restricting the bundle $E^{*}$ into a trivializing open subset $U \subseteq Z$ such that $E_{U}^{*} \simeq \mathbb{C}^{r} \otimes \mathcal{O}_{U}$ where $\mathbb{C}^{r}$ is spanned by $e_{1}, \ldots, e_{r}$. We can suppose that, with respect of this frame, $s=\left(s_{1}, \ldots, s_{r}\right)$ and that $Z^{\prime} \cap U$ is the zero locus of $s^{\prime}=\left(s_{1}, \ldots, s_{r^{\prime}}\right)$.

Let us proceed by induction on $r-r^{\prime}$. If $r=r^{\prime}$, then $Z_{0}$ is a complete intersection and the Koszul complex is exact ([GH], p. 688).

Let us suppose now $r>r^{\prime}$ and let $E_{k}=\wedge^{k} E_{U}^{*}$ and $F_{k}=\wedge^{k} \mathbb{C}^{r-1} \otimes$ $\mathcal{O}_{U} \subseteq \wedge^{k} \mathbb{C}^{r} \otimes \mathcal{O}_{U} \simeq E_{k}$. The quotient $Q_{k}$ of $E_{k}$ by $F_{k}$ is isomorphic to $\left(e_{r} \otimes \wedge^{k-1} \mathbb{C}^{r-1}\right) \otimes \mathcal{O}_{U}$, moreover the map $\delta: E_{k} \rightarrow E_{k-1}$ induces, in a canonical way, two maps $\delta^{\prime}: F_{k} \rightarrow F_{k-1}$ and $\delta^{\prime \prime}: Q_{k} \rightarrow Q_{k-1}$ so that $F_{*}$ and $Q_{*}$ are again Koszul complexes contained in the commutative diagram:

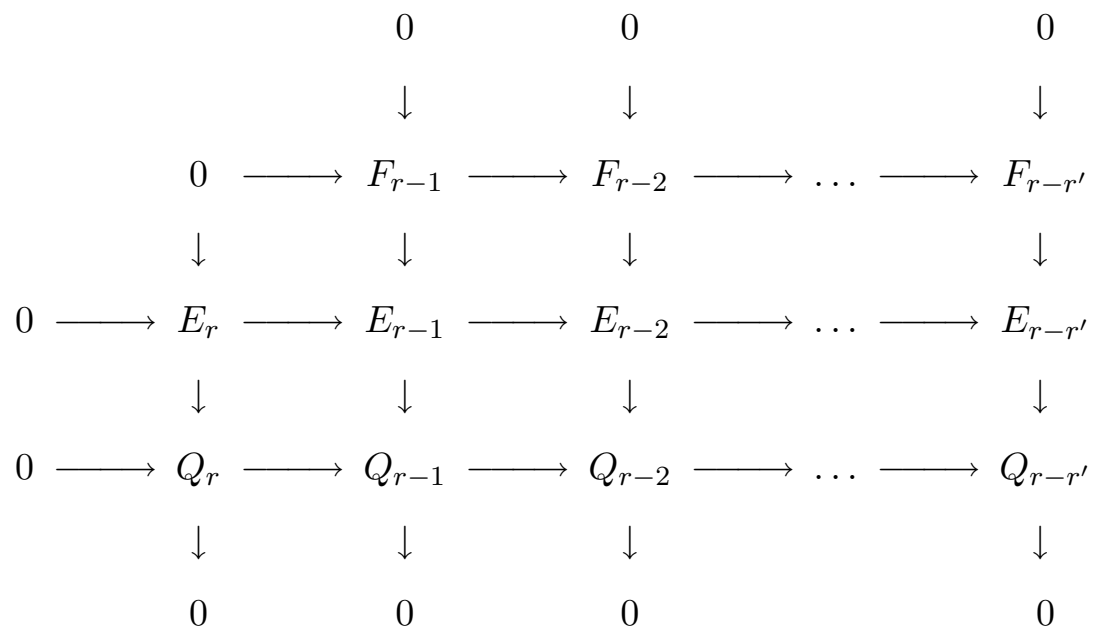

By induction hypothesis, $\mathrm{H}_{*}\left(F_{*}\right)=\mathrm{H}_{*}\left(Q_{*}\right)=0$. Thus also $\mathrm{H}_{*}\left(E_{*}\right)=0$, i.e., the sequence (3) is exact.

Lemma 3.6. For any $r=1, \ldots, m-1$, the sheaf $\wedge^{r} \mathcal{F}_{A}$ is contained in the exact sequence:

$$
I^{*} \otimes \wedge^{r-1} W^{*} \otimes \mathcal{O}_{\mathbb{P}^{n}}(r-1) \rightarrow \wedge^{r} W^{*} \otimes \mathcal{O}_{\mathbb{P}^{n}}(r) \rightarrow \wedge^{r} \mathcal{F}_{A} \rightarrow 0 .
$$


Moreover, if $A \in X$ is G.I.T. stable and $r \leq \frac{m-1}{2}$ then the sequence

$$
\begin{aligned}
0 & \rightarrow S^{r} I^{*} \otimes \mathcal{O}_{\mathbb{P}^{n}} \rightarrow S^{r-1} I^{*} \otimes W^{*} \otimes \mathcal{O}_{\mathbb{P}^{n}}(1) \rightarrow \ldots \\
& \ldots \rightarrow I^{*} \otimes \wedge^{r-1} W^{*} \otimes \mathcal{O}_{\mathbb{P}^{n}}(r-1) \rightarrow \wedge^{r} W^{*} \otimes \mathcal{O}_{\mathbb{P}^{n}}(r) \rightarrow \wedge^{r} \mathcal{F}_{A} \rightarrow 0
\end{aligned}
$$

is exact. In particular, $\operatorname{hd}\left(\wedge^{r} \mathcal{F}_{A}\right) \leq r$.

Proof. The exactness of (4) is proven in ([Eis], p. 571).

In order to prove the exactness of (5), we proceed by mimicking the proof of the existence of the Eagon-Northcott complex given in [GP].

Let $Z=\mathbb{P}(V) \times \mathbb{P}\left(I^{*}\right)$ and let $\pi: Z \rightarrow \mathbb{P}(V)$ be the projection onto the first space. The morphism $A$ defines a section $a: \mathcal{O}_{Z} \rightarrow W^{*} \otimes \mathcal{O}_{Z}(1,1)$, given by $a=\left(y_{0} f_{0, i}+y_{1} f_{1, i}\right)_{i=1}^{m+2}$ where the $f_{i, j}$ 's are the entries of $A$ and $y_{0}, y_{1}$ are the coordinates of $\mathbb{P}\left(I^{*}\right)$.

The zero locus of $a$ is $\widetilde{Z}=\cap_{i} V\left(y_{0} f_{0, i}+y_{1} f_{1, i}\right) \subseteq Z$, and the Koszul complex associated is given by:

$$
\begin{aligned}
& \rightarrow \wedge^{m+2} W \otimes \mathcal{O}_{Z}(-m-2,-m-2) \rightarrow \ldots \\
\cdots & \rightarrow \wedge^{2} W \otimes \mathcal{O}_{Z}(-2,-2) \rightarrow W \otimes \mathcal{O}_{Z}(-1,-1) \rightarrow \mathcal{O}_{Z} \rightarrow \mathcal{O}_{\widetilde{Z}} \rightarrow 0 .
\end{aligned}
$$

We have that $\pi(\widetilde{Z}) \subseteq D(A)$ and, since $A$ is stable, then $\operatorname{dim} \widetilde{Z} \leq \operatorname{dim} D(A)+$ $1 \leq n-\frac{m-1}{2}$ (Cor. 2.4).

By Lemma 3.5, the sequence:

$$
0 \rightarrow \wedge^{m+2} W \otimes \mathcal{O}_{Z}(-r-2,-r-2) \rightarrow \cdots \rightarrow \wedge^{m+2-r} W \otimes \mathcal{O}_{Z}(-2,-2),
$$

is exact for any $r \leq \frac{m-1}{2}$.

Since each fiber of $\pi$ is isomorphic to $\mathbb{P}\left(I^{*}\right)$, it results in:

$$
R^{i} \pi_{*}\left(\mathcal{O}_{Z}(-2-j,-2-j)\right)= \begin{cases}S^{j} I^{*} \otimes \mathcal{O}_{\mathbb{P}^{n}}(-2-j) & \text { if } i=1 \quad j \geq 0 \\ 0 & \text { if } i \neq 1 \quad j \geq 0\end{cases}
$$

where $R^{i} \pi_{*}$ is the higher direct image functor associated to $\pi$ (see [Har], Ch. III, 8). Moreover $\wedge^{m+2-r+j} W \simeq \wedge^{r-j} W^{*}$, that yields the exact sequence:

$$
0 \rightarrow S^{r} I^{*} \otimes \mathcal{O}_{\mathbb{P}^{n}}(-r-2) \rightarrow \cdots \rightarrow \wedge^{r} W^{*} \otimes \mathcal{O}_{\mathbb{P}^{n}}(-2) .
$$

The exactness of the sequence (5) follows by gluing (6) tensored by $\mathcal{O}_{\mathbb{P}^{n}}(r+2)$ with (4): In fact, in both the sequences the morphisms

$$
I^{*} \otimes \wedge^{r-1} W^{*} \otimes \mathcal{O}_{\mathbb{P}^{n}}(r-1) \rightarrow \wedge^{r} W^{*} \otimes \mathcal{O}_{\mathbb{P}^{n}}(r)
$$

are canonically defined.

Lemma 3.7. Let $E$ be a coherent sheaf and $S(E)$ its singular locus. If codim $S(E) \geq \operatorname{hd}(E)+2$, then $E$ is reflexive. 
Proof. Let $t=\mathrm{hd}(E)$ and let us consider a resolution of $E$ :

$$
0 \rightarrow F_{t} \rightarrow F_{t-1} \rightarrow F_{t-2} \rightarrow \cdots \rightarrow F_{2} \rightarrow F_{1} \rightarrow F_{0} \rightarrow E \rightarrow 0
$$

where each $F_{i}$ is a direct sum of line bundles.

Let us split the sequence into short exact sequences:

$$
\begin{gathered}
0 \rightarrow F_{t} \rightarrow F_{t-1} \rightarrow G_{t-1} \rightarrow 0 \\
0 \rightarrow G_{i} \rightarrow F_{i-1} \rightarrow G_{i-1} \rightarrow 0 \\
0 \rightarrow G_{1} \rightarrow F_{0} \rightarrow E \rightarrow 0
\end{gathered}
$$

then, applying the functor $\mathcal{E} x t^{i}\left(\cdot, \omega_{\mathbb{P} n}\right)$, we get:

$$
\mathcal{E} x t^{i}\left(E, \omega_{\mathbb{P}^{n}}\right)=\mathcal{E} x t^{i-1}\left(G_{1}, \omega_{\mathbb{P}^{n}}\right)=\cdots=\mathcal{E} x t^{i-t+1}\left(G_{t-1}, \omega_{\mathbb{P}^{n}}\right)=0,
$$

for any $i$ such that $i-t+1 \geq 2$. Thus $\mathcal{E} x t^{i}\left(E, \omega_{\mathbb{P} n}\right)=0$ for any $i \geq t+1$.

Moreover

$$
\operatorname{codim} \mathcal{E} x t^{i}\left(E, \omega_{\mathbb{P}^{n}}\right) \geq \operatorname{codim} S(E) \geq t+2 \geq i+2
$$

if $1 \leq i \leq t$ and thus by Proposition 3.4, $E$ is reflexive.

Lemma 3.8. Let $E$ be a sheaf of rank $m$ on $\mathbb{P}^{n}$ such that $\operatorname{codim} S(E) \geq 2$. Then, for any $r=1, \ldots, m-1$, we have

$$
\left(\wedge^{r} E\right)^{* *}=\left(\wedge^{m-r} E\right)^{*} \otimes \mathcal{O}_{\mathbb{P}^{n}}\left(c_{1}(E)\right) .
$$

Proof. The injective map $\wedge^{m-r} E \rightarrow \operatorname{Hom}\left(\wedge^{r} E, \wedge^{m} E\right)$ induces the exact sequence:

$$
0 \rightarrow \wedge^{m-r} E \rightarrow \operatorname{Hom}\left(\wedge^{r} E, \wedge^{m} E\right) \rightarrow \mathcal{E} \rightarrow 0,
$$

where $\mathcal{E}$ is a 0 -rank sheaf such that $\operatorname{codim} \mathcal{E} \geq \operatorname{codim} S(E) \geq 2$ : Thus, dualizing this sequence and observing that, by Proposition $3.3, \mathcal{E}^{*}=\mathcal{E} x t^{1}\left(\mathcal{E}, \mathcal{O}_{\mathbb{P}} n\right)=$ 0 , it results $\left(\wedge^{m-r} E\right)^{*} \simeq \operatorname{Hom}\left(\wedge^{r} E, \wedge^{m} E\right)^{*} \simeq\left(\wedge^{r} E\right)^{* *} \otimes\left(\wedge^{m} E\right)^{*}$, in fact by Proposition 3.4 all these sheaves are torsion-free. Moreover $\left(\wedge^{m} E\right)^{* *} \simeq$ $\mathcal{O}_{\mathbb{P}^{n}}\left(c_{1}(E)\right)$ and therefore $(7)$ follows.

Thus it results $\mathrm{H}^{0}\left(\left(\wedge^{r} \mathcal{F}_{A}\right)_{N}^{* *}\right)=\mathrm{H}^{0}\left(\left(\wedge^{m-r} \mathcal{F}_{A}\right)^{*}\left(t_{0}\right)\right)$ for suitable $t_{0} \in \mathbb{Z}$ : We want to prove that such cohomology group is null.

Let us distinguish 2 cases:

I. $r \geq \frac{m+1}{2}$ :

Let $t_{0}$ be such that $\left(\wedge^{r} \mathcal{F}_{A}\right)_{N}^{* *}=\left(\wedge^{m-r} \mathcal{F}_{A}\right)^{*}\left(t_{0}\right)$. By the sequence $(4)$, we have:

$$
0 \rightarrow\left(\wedge^{m-r} \mathcal{F}_{A}\right)^{*}\left(t_{0}\right) \rightarrow \wedge^{m-r} W \otimes \mathcal{O}_{\mathbb{P}^{n}} \stackrel{B}{\longrightarrow} \wedge^{m-r-1} W \otimes I^{\otimes} \mathcal{O}_{\mathbb{P}^{n}}(1) .
$$

Thus if $\mathrm{H}^{0}\left(\left(\wedge^{m-r} \mathcal{F}_{A}\right)^{*}\left(t_{0}\right)\right) \neq 0$ then there exists $b: \mathcal{O}_{\mathbb{P}} \hookrightarrow \wedge^{m-r} W \otimes \mathcal{O}_{\mathbb{P} n}$ (i.e., $b \in \wedge^{m-r} W$ ) such that $B \circ b=0$.

It is easy to see that if $A$ is injective then $B \circ b=0$ implies $b=0$.

II. $r \leq \frac{m-1}{2}$ : 
If $4 \operatorname{codim} D(A) \geq 2+\frac{m-1}{2}=\frac{m+3}{2}$, then, by Lemma 3.7, the sheaf $\wedge^{r} \mathcal{F}$ is reflexive, and by the sequence (5), it is easy to show that:

$$
\mathrm{H}^{0}\left(\left(\wedge^{r} \mathcal{F}_{A}\right)_{N}^{* *}\right)=\mathrm{H}^{0}\left(\left(\wedge^{r} \mathcal{F}_{A}\right)_{N}\right)=0 .
$$

By Corollary 2.4, we have that the G.I.T. stability of $A$ implies that codim $D(A) \geq \frac{m+1}{2}$ and thus it just remains to consider the matrices $A$ such that $\operatorname{codim} D(A)=\frac{m+1}{2}$.

Lemma 3.9. If $\operatorname{codim} D(A)=\frac{m+1}{2}$ then $\mathrm{H}^{0}\left(\left(\wedge^{r} \mathcal{F}_{A}\right)_{N}^{* *}\right)=0$ for any $r=$ $1, \ldots, m-1$.

Proof. By Proposition 2.5, we can suppose

$$
f_{A}=\left(\begin{array}{ccccccc}
x_{0} & \ldots & x_{t-1} & 0 & \ldots & 0 & x_{t} \\
0 & \ldots & 0 & x_{0} & \ldots & x_{t-1} & x_{t+1}
\end{array}\right)^{t} .
$$

Moreover the same technique used above can be applied to prove the thesis for all $r \neq t$.

Thus it suffices to show that $\mathrm{H}^{0}\left(\left(\wedge^{t} \mathcal{F}_{A}\right)^{*}\left(t_{0}\right)\right)=\mathrm{H}^{0}\left(\left(\wedge^{t-1} \mathcal{F}_{A}\right)_{N}^{* *}\right)=0$. It is easily checked that by dualizing the sequence $(5)$ we get the sequence

$$
0 \rightarrow\left(\wedge^{t} \mathcal{F}_{A}\right)^{*}\left(t_{0}\right) \rightarrow \wedge^{t} W \otimes \mathcal{O}_{\mathbb{P}^{n}}(1) \stackrel{B}{\longrightarrow} \wedge^{t-1} W \otimes I \otimes \mathcal{O}_{\mathbb{P}^{n}}(2) .
$$

Thus we just need to prove that if $b: \mathcal{O}_{\mathbb{P}^{n}} \rightarrow \wedge^{t} W \otimes \mathcal{O}_{\mathbb{P}^{n}}(1)$ is such that $B \circ b=0$ then $b=0$ : This is a direct computation.

Thus Lemma 3.2 is completely proven. We can proceed now with the Proof of Theorem 3.1.

Proof of Theorem 3.1. The first statement of Proposition 3.4 easily implies that the map $\mathcal{F}_{A} \rightarrow\left(\mathcal{F}_{A}\right)^{* *}$ is injective. Since $\left(\mathcal{F}_{A}\right)^{* *}$ is torsion-free, so is $\mathcal{F}_{A}$.

Let now $\mathcal{E} \subseteq \mathcal{F}_{A}$ be a torsion-free sub-sheaf of rank $r$. Then $\mathcal{O}_{\mathbb{P} n}\left(c_{1}(\mathcal{E})\right)=$ $\left(\wedge^{r} \mathcal{E}\right)^{* *} \subseteq\left(\wedge^{r} \mathcal{F}_{A}\right)^{* *}$ : Since $\mathrm{H}^{0}\left(\left(\wedge^{r} \mathcal{F}_{A}\right)_{N}^{* *}\right)=0$ (Lemma 3.2), it results $c_{1}(\mathcal{E})<\mu\left(\wedge^{r} \mathcal{F}_{A}\right)=r \mu\left(\mathcal{F}_{A}\right)$, i.e., $\mu(\mathcal{E})<\mu\left(\mathcal{F}_{A}\right)$. Thus $\mathcal{F}_{A}$ is $\mu$-stable.

Vice-versa, let $A \in X$ be a non-stable matrix. Then, by Theorem 1.1, we can write $A=\left(\begin{array}{cc}0 & A_{0} \\ A_{1} & A_{2}\end{array}\right)$ where $A_{0}$ is a vector of length $s \leq \frac{m+1}{2}$. Thus $A_{0}$ defines a sub-sheaf $\mathcal{F}_{A_{0}} \subseteq \mathcal{F}_{A}$ that is contained in the exact sequence:

$$
0 \longrightarrow \mathcal{O}_{\mathbb{P}^{n}} \stackrel{f_{A_{0}}}{\longrightarrow} \mathcal{O}_{\mathbb{P}^{n}}(1)^{s} \longrightarrow \mathcal{F}_{A_{0}} \longrightarrow 0 .
$$

It is easily checked that $\mu\left(\mathcal{F}_{A_{0}}\right)>\mu\left(\mathcal{F}_{A}\right)$.

We show now an interesting relation within the automorphism group of $\mathcal{F}_{A}$ and the stabilizer of $A$. 
Theorem 3.10. Let $A \in X$ such that $\mathcal{F}_{A}$ is simple, i.e., $\operatorname{Aut}\left(\mathcal{F}_{A}\right)=\mathbb{C}^{*}$. Then

$$
\operatorname{Stab}_{G}(A)=\left\{\left(\lambda \operatorname{Id}_{2}, \mu \operatorname{Id}_{m+2}\right) \in G \mid \lambda^{n+k}=\mu^{k}=1\right\} .
$$

In particular $\operatorname{dim} \operatorname{Stab}_{G}(A)=0$ and $\operatorname{dim} \mathcal{M}_{n, m, 2}=\operatorname{dim} X-\operatorname{dim} G$, for any $m<2 n$.

Proof. Let us prove first that any $f \in \operatorname{Aut}\left(\mathcal{F}_{A}\right)$ is uniquely determined by a morphism of sequences:

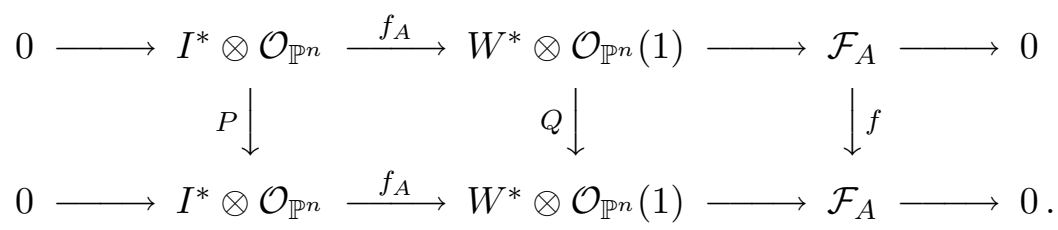

This is a direct consequence of the fact that, by the vanishing of $\operatorname{Hom}\left(\mathcal{O}_{\mathbb{P}^{n}}^{n+k}, \mathcal{O}_{\mathbb{P}^{n}}(-1)^{k}\right)$ and $\operatorname{Ext}^{1}\left(\mathcal{O}_{\mathbb{P}^{n}}^{n+k}, \mathcal{O}_{\mathbb{P}^{n}}(-1)^{k}\right)$ we get the exact sequence:

$$
0 \rightarrow \operatorname{Hom}\left(\mathcal{O}_{\mathbb{P}^{n}}^{n+k}, \mathcal{O}_{\mathbb{P}^{n}}^{n+k}\right) \rightarrow \operatorname{Hom}\left(\mathcal{O}_{\mathbb{P}^{n}}^{n+k}, \mathcal{F}_{A}\right) \rightarrow 0 .
$$

Thus if $\mathcal{F}_{A}$ is simple, then the only automorphisms of $\mathcal{F}_{A}$ are the homotheties, that implies (8).

We are ready now to prove Theorem 1.2. Let $c_{1}, \ldots, c_{n}$ be the Chern classes of $\mathcal{F}_{A}$ and let $\mathcal{M}_{\mathbb{P}^{n}}\left(m, c_{1}, \ldots, c_{n}\right)$ be the Maruyama moduli space of all the $\mu$-stable sheaves or rank $m$ and Chern classes $c_{1}, \ldots, c_{n}$. By Theorem 3.1, if $m$ is odd, each $A \in \mathcal{M}_{n, m, 2}$ defines uniquely an isomorphism class of coherent sheaves $\left[\mathcal{F}_{A}\right] \in \mathcal{M}_{\mathbb{P}^{n}}\left(m, c_{1}, \ldots, c_{n}\right)$ and thus there exists an injective projective morphism:

$$
\phi: \mathcal{M}_{n, m, 2} \longrightarrow \mathcal{M}_{\mathbb{P}^{n}}\left(m, c_{1}, \ldots, c_{n}\right) .
$$

Moreover, by the sequence (1) that defines $\mathcal{F}_{A}$, it is easily checked that $\operatorname{Ext}^{2}\left(\mathcal{F}_{A}, \mathcal{F}_{A}\right)=0$, i.e., every point of the image of $\phi$ is a smooth point of the Maruyama moduli space. By Theorem 3.10 and by sequence (1), it immediately follows that $\operatorname{dim} \mathcal{M}_{n, m, 2}=\operatorname{dim} \operatorname{Ext}^{1}\left(\mathcal{F}_{A}, \mathcal{F}_{A}\right)=\operatorname{dim}_{\left[\mathcal{F}_{A}\right]} \mathcal{M}_{\mathbb{P}^{n}}\left(m, c_{1}\right.$, $\left.\ldots, c_{n}\right)$, and thus, by Stein factorization theorem, $\phi$ maps isomorphically $\mathcal{M}_{n, m, 2}$ onto a smooth connected component of $\mathcal{M}_{\mathbb{P}^{n}}\left(m, c_{1}, \ldots, c_{n}\right)$. This completely proves Theorem 1.2.

Remark 3.11. In general, the same result does not hold for higher $k$, even if $(m, k)=1$ (i.e., in the case where all the semi-stable sheaves are stable). Consider, for istance, the matrix

$$
f=\left(\begin{array}{ccccc}
x_{1} & x_{0} & 0 & 0 & 0 \\
0 & x_{2} & x_{1} & x_{0} & 0 \\
0 & 0 & 0 & x_{2} & x_{1}
\end{array}\right)^{t}
$$


and the sequence

$$
0 \longrightarrow \mathcal{O}_{\mathbb{P}^{2}}^{3} \stackrel{f}{\longrightarrow} \mathcal{O}_{\mathbb{P}^{2}}(1)^{5} \longrightarrow \mathcal{F}_{A} \longrightarrow 0 .
$$

Then, since the degeneracy locus of the sheaf $\mathcal{F},\left\{x_{1}=0\right\}$, is of codimension $1, \mathcal{F}$ is not torsion-free and in particular it cannot be $\mu$-stable. On the other hand, using Theorem 1.1, it is a direct computation to prove that $f$ defines a stable morphism.

\section{References}

[AO] V. Ancona and G. Ottaviani, Unstable hyperplanes for Steiner bundles and multidimensional matrices, Adv. Geom., 1(2) (2001), 165-192, CMP 1840220.

[C] P. Cascini, On a compactification of the moduli space of the rational normal curves, preprint, math.AG/9912070.

[DK] I. Dolgachev and M. Kapranov, Arrangement of hyperplanes and vector bundles on $\mathbb{P}^{n}$, Duke Math. J., 71 (1993), 633-664, MR 95e:14029, Zbl 0804.14007.

[Dr1] J.-M. Drezet, Fibrés exceptionnels et variétés de modules de fasceaux semistables sur $\mathbb{P}^{2}(\mathbb{C})$, J. Reine Angew. Math., 380 (1987), 14-58, MR 89e:14016, Zbl 0613.14013.

[Dr2] Cohomologie des variétés de modules de hauteur nulle, Math. Ann., 281 (1988), 43-85, MR 89j:14009, Zbl 0644.14005.

[Dr3] _ Exceptional bundles and moduli spaces of stable sheaves on $\mathbb{P}^{n}$, Lond. Math. Soc. Lect. Note Ser., 208 (1995), 101-117, MR 96i:14033, Zbl 0860.14018.

[Eis] D. Eisenbud, Commutative Algebra with a View Toward Algebraic Geometry, Graduate Texts in Math. 151, Springer, 1995, MR 97a:13001, Zbl 0819.13001.

[EPS] G. Ellingsrud, R. Piene and S.A. Strømme, On the variety of nets of quadrics defining twisted cubic curves, in F. Ghione, C. Peskine, E. Sernesi, 'Space Curves,' Lecture Notes in Mathematics, 1266, Springer, 1987, 84-96, MR 88h:14034, Zbl 0659.14027.

[ES] G. Ellingsrud and S.A. Strømme, On the Chow ring of a geometric quotient, Ann. Math., 130 (1989), 159-187, MR 90h:14019, Zbl 0716.14002.

[GKZ] I.M. Gelfand, M.M. Kapranov and A.V. Zelevinsky, Discriminants, Resultants and Multidimensional Determinants, Birkhäuser, Boston, 1994, MR 95e:14045, Zbl 0827.14036.

[GH] P. Griffiths and J. Harris, Principles of Algebraic Geometry, John Wiley and Sons, New York (1978), MR 80b:14001, Zbl 0408.14001.

[GP] L. Gruson and C. Peskine, Courbes de l'espace projectif: Varietes de secantes, Enumerative geometry and classical algebraic geometry, Prog. Math., 24 (1982), 1-31, MR 84m:14061, Zbl 0531.14020.

[Har] R. Hartshorne, Algebraic Geometry, Graduate Texts in Math., 52, Springer, 1977, MR 57 \#3116, Zbl 0367.14001.

[HL] D. Huybrechts and M. Lehn, The Geometry of Moduli Space of Sheaves, Aspects of Mathematics, 31, Vieweg, Braunschweig, 1997, MR 98g:14012, Zbl 0872.14002. 
[MT] R.M. Miró-Roig and G. Trautmann, The moduli scheme $\mathcal{M}(0,2,4)$ over $\mathbb{P}^{3}$, Math. Z., 216 (1994), 283-215, MR 95i:14016, Zbl 0837.14008.

[MFK] D. Mumford, J. Fogarty and F.Kirwan, Geometric Invariant Theory, third enlarged edition, Ergebnisse der Mathematik und ihrer Grenzgebiete, 34, Springer, 1994, MR 95m:14012, Zbl 0797.14004.

[OSS] C. Okonek, M. Schneider and H. Spindler, Vector Bundles on Complex Projective Space, Birkhäuser, Basel and Boston, Mass., 1980, MR 81b:14001, Zbl 0438.32016.

[Schw] R.L.E. Schwarzenberger, Vector bundles on the projective plane, Proc. London Math. Soc., 11 (1961), 623-640, MR 25 \#1161, Zbl 0212.26004.

[V] J. Vallès, Nombre maximal d'hyperplans instables pour un fibré de Steiner, Math. Z., 233 (2000), 507-514, MR 2001e:14040, Zbl 0952.14011.

Received August 252000 and revised April 6, 2001.

Courant Institute of Mathematical Sciences

NeW York University

25 Mercer ST.

NEW York, NY 10012

E-mail address: cascini@cims.nyu.edu 